\title{
Résultats et perspectives de la radioprotection des travailleurs sur un centre de stockage des déchets de faible et moyenne activité $\left({ }^{\star}\right)$
}

\author{
J. SCHEIDHAUER et CI. LASSEUR (**) \\ (Manuscrit reçu le 12 octobre 1981)
}

\begin{abstract}
RÉSUMÉ
Après une présentation rapide du Centre Manche (CM) et de son histoire, les auteurs rappellent les diverses règles ou règlements et montrent leur incidence sur le fonctionnement du centre. Ils définissent les sources de rayonnements puis les différents postes de travail que présente un centre national de stockage des déchets de faible et moyenne activité. Ils analysent les problèmes de radioprotection liés à chacun des postes : organisation, prévention, protection collective et individuelle, dosimétrie. Ils présentent les résultats dosimétriques de plus de dix ans d'exploitation. Ils discutent des différents moyens de réduire les expositions du personnel d'exploitation, en particulier l'incidence des normes de transport.
\end{abstract}

\section{ABSTRACT}

The national low- and intermediate-level waste storage center (CM) located near the Channel is presented, together with its history, the various regulations governing it and their incidence on its operation. A description is given of the radiation sources and workplaces to be found in this kind of waste storage center. The radiation protection problems associated with the respective workplaces are analysed: organization, prevention, collective and individual protection, dosimetry. Occupational radiation exposures from 1970 to 1980 are presented. The various means of reducing operating workers' exposures are discussed, especially the incidence of transportation.

Le stockage des déchets de faible et moyenne activité, sur un centre non annexé à un ou plusieurs établissements producteurs, est certainement le dernier né de la famille des établissements nucléaires ou plus précisément des installations nucléaires de base (INB).

(*) Communication présentée lors du séminaire sur les "Progrès dans les techniques de radioprotection", Cadarache, 5-7 mai 1981.

(*) STMI, Val Courcelle, 9, rue Fernand Léger, 91190 Gif-sur-Yvette. 
Ce type d'installations regroupant certains conditionnements et le stockage des déchets n'est, en général, pas identifié en origine de production, particulièrement en ce qui concerne la protection des travailleurs contre les rayonnements ionisants.

Le cas du Centre de stockage de la Manche (CM), dont l'exploitant nucléaire, au sens de la loi, est le Commissariat à l'énergie atomique, qui en a confié la responsabilité à l'Agence nationale pour la gestion des déchets radioactifs (ANDRA) est, en ce sens, exemplaire. II convient, avant tout, de présenter l'histoire de cet établissement pour situer l'action de notre société, et pour bien montrer que l'expérience tirée de cette exploitation a été continue et a permis des observations intéressantes.

Le centre de stockage a été ouvert en janvier 1969 par le CEA, qui confie l'exploitation à la société Infratome, filiale de PEC Engineering. Ultérieurement, Infratome est intégré à la société mère sous forme de département. Le Commissariat dénonce pour compte du 1er novembre 1979 le contrat le liant à PEC Engineering. II confie alors l'exploitation technique du Centre à la Société de travaux en milieu ionisant (STMI) en attente de la création de la Société industrielle de stockage et d'assainissement (SISA) qui intervient le 3 juillet 1980. Au terme de l'article L 122 du Code du travail, l'ensemble du personnel d'exploitation est repris par la STMI. Le 1er janvier 1981, l'ANDRA confie le contrat d'exploitation technique à la SISA qui en effectue la gestion mais qui en confie l'exécution à la STMI.

Le même personnel poursuit donc son activité à travers toutes les évolutions contractuelles. Ceci permet une analyse significative de son activité vis-à-vis de la protection contre les rayonnements. Cette analyse va procéder successivement par: l'examen de la réglementation, les sources de rayonnements, les postes de travail et les expositions associées, les résultats dosimétriques et leur analyse, les tendances et perspectives.

Enfin, précisons que les déchets de faible et moyenne activité arrivent au CM uniquement par la route, soit en transconteneur, soit en emballage de transport, soit en coque, soit en blocs. Le transport en transconteneur se fait par lot complet de fûts de 220, 200 ou 100 litres. De même, certains emballages à coques multiples contiennent plusieurs fûts de 50 ou 60 litres (28 fûts pour les emballages RD 12, 14 fûts pour les emballages RD 11). Chaque élément, constituant du contenu, est appelé un colis. Tout colis entrant au CM doit être un colis préalablement agréé par l'ANDRA conformément au catalogue des colis agréés [1].

\section{LA RÉGLEMENTATION}

La collecte et le transport des déchets radioactifs doivent être assurés dans le respect de l'ensemble des textes législatifs et règlements constitués par: les décrets et arrêtés concernant la protection des travailleurs et des personnes du public contre les rayonnements ionisants [2-4], la réglementation de transport des matières radioactives (classe IV b), arrêté du 24 juin 1974 modifié et codifié à la date du 8 août 1980 [5], les règles d'agrément des colis contenant des déchets radioactifs émises par l'ANDRA (catalogue des colis agréés) [1]. 
II n'est pas dans notre propos de présenter dans le détail ces textes et ces règles mais d'en tirer les éléments principaux.

\section{I.1. Décrets et arrêtés concernant la protection contre les rayonnements ionisants}

Le Centre de stockage de la Manche (CM) est une installation nucléaire de base.II est donc soumis au décret du 28 avril 1975 [3], relatif à la protection des travailleurs contre les dangers des rayonnements ionisants dans les INB et aux arrêtés d'application de juillet et octobre 1977 [4]. Ces divers textes imposent des mesures d'ordre administratif et technique dont les principales sont :

- la préparation au travail sous rayonnements et la remise d'une notice d'informations;

- l'affichage du règlement intérieur;

- la délimitation et la signification de la zone contrôlée ainsi que des zones spécialement réglementées ou interdites à l'intérieur de la zone contrôlée ;

- le contrôle périodique des dispositifs de protection, de détection des rayonnements, de signalisation et d'alarme;

- la surveillance individuelle de l'irradiation et de la contamination pour les travailleurs directement affectés à des travaux sous rayonnements.

\section{I.2. Le règlement de transport}

Du règlement de transport, nous ne retiendrons que la limitation des rayonnements s'échappant de l'emballage :

- produits de la $4^{e}$ catégorie (groupes 42401, 42402, 42403, 42404) $5 \mu \mathrm{Sv} . \mathrm{h}^{-1}$ (ou son équivalent) au contact de l'emballage ;

- produits des $1^{\mathrm{re}}, 2^{\mathrm{e}}$ et $3^{\mathrm{e}}$ catégories : $2 \mathrm{mSv}^{-1} \mathrm{~h}^{-1}$ (ou son équivalent) au contact de l'emballage, $0,1 \mathrm{mSv} \cdot \mathrm{h}^{-1}$ (ou son équivalent) à 1 mètre de la surface extérieure de l'emballage.

Il est fixé, en outre, une limitation des rayonnements s'échappant du véhicule ou du wagon : $10 \mathrm{mSv} \cdot \mathrm{h}^{-1}$ (ou son équivalent) à la surface extérieure du colis à condition que le véhicule ou le wagon soit fermé de façon à empêcher toute intervention entre le début et la fin du transport et à assurer la stabilité du chargement ; $2 \mathrm{mSv}^{\mathrm{h}} \mathrm{h}^{-1}$ (ou son équivalent) au contact de la surface extérieure du véhicule ou du wagon ; $0,10 \mathrm{mSv} \cdot \mathrm{h}^{-1}$ (ou son équivalent) à $2 \mathrm{~m}$ des surfaces latérales externes du véhicule ou du wagon.

Ces limites assurent une très bonne protection pour le personnel affecté au transport et pour la population. Nous examinerons plus loin les contraintes induites sur le personnel d'exploitation du CM.

\section{I.3. Le catalogue des colis agréés}

Ce catalogue est à la fois une règle imposant certains colis, c'est-à-dire le conditionnement et le conteneur primaire et une tolérance en admettant certains autres à titre transitoire. Ne seront admis sur le CM que les colis figurant au catalogue qui intègre les prescriptions du Service central de sureté des installations nucléaires pour le CM. Ces règles définissent essentiellement la nature 


\section{J. SCHEIDHAUER, C. LASSEUR}

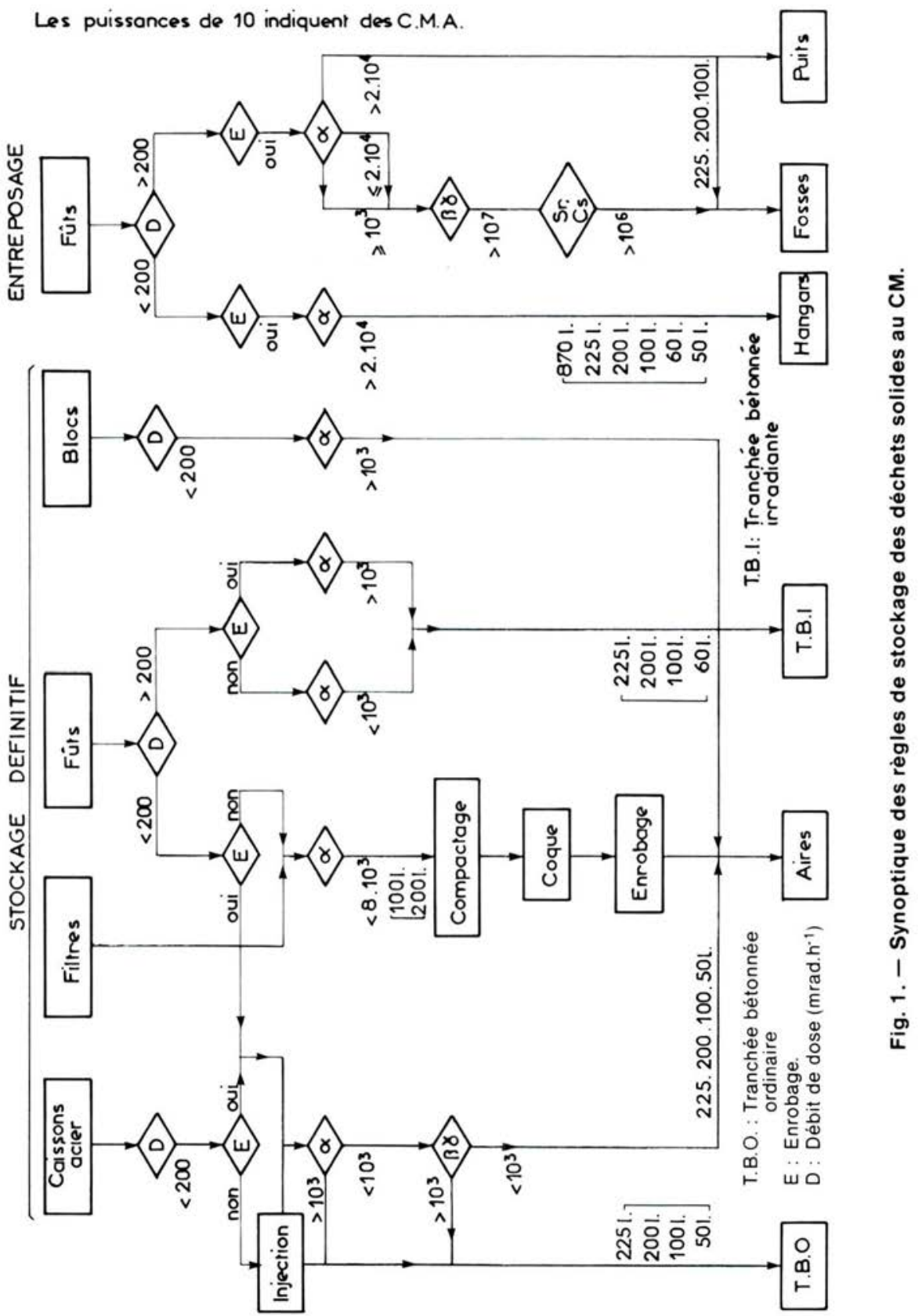


des radionucléides et l'activité spécifique du déchet, associées à un conditionnement précis et à des débits de dose reprenant, pour une grande part, les débits d'équivalent de dose retenus par la réglementation des transports (fig. 1). Ce catalogue est une pièce contractuelle liant le producteur de déchets et l'agence. II constitue un document réglementaire pour l'opérateur effectuant la prise en charge des déchets au nom de l'ANDRA.

\section{LES SOURCES DE RAYONNEMENTS}

Une source radioactive est, en général, caractérisée par sa nature, sa forme et ses dimensions, son activité, sa période, etc. Les sources rencontrées au CM sont de natures variées mais on peut les classer en deux grandes catégories: les colis reçus ou sources statiques, les structures de stockage ou sources dynamiques.

\section{II.1. Les types de sources (fig. 2)}

\section{II.1.1. Les sources statiques}

Chaque colis reçu constitue une source volumique élémentaire dont le conditionnement va subir ou non diverses transformations avant la dépose en structure de stockage. Ces colis proviennent de deux origines:

- Des producteurs non nucléaires appelés encore "petits producteurs" qui sont les centres de recherche, les universités et laboratoires, les hôpitaux et centres de soins spécialisés et les industries diverses. Ces producteurs relèvent de la réglementation gouvernant l'utilisation des radio-éléments (sources scellées et non scellées). Le volume de ces déchets a atteint $1000 \mathrm{~m}^{3}$ en 1980.

- Des producteurs nucléaires qui sont les établissements industriels du cycle du combustible nucléaire, les centrales nucléaires, et les centres d'études nucléaires. Les déchets produits y sont de deux types, les déchets technologiques et les déchets de procédés ou spécifiques. Ces producteurs relèvent de la réglementation relative aux installations nucléaires de base ou INB.

\subsubsection{Les sources dynamiques}

Le stockage des sources statiques précédentes se fait selon deux principes :

- un stockage définitif sur aire ou en tranchée bétonnée (TB) ;

- un entreposage en hangars, fosses ou puits, en vue d'une reprise possible ou obligatoire.

Chaque structure constitue donc une source de grand volume dont les caractéristiques se modifient avec l'état d'avancement (aire et TB) ou avec le remplissage (hangar, fosse, puits). Notons que, le stockage réalisé, la source ne doit pratiquement plus contribuer à l'irradiation des travailleurs.

En résumé, les sources rencontrées sur le CM peuvent se schématiser ainsi (fig. 2): 


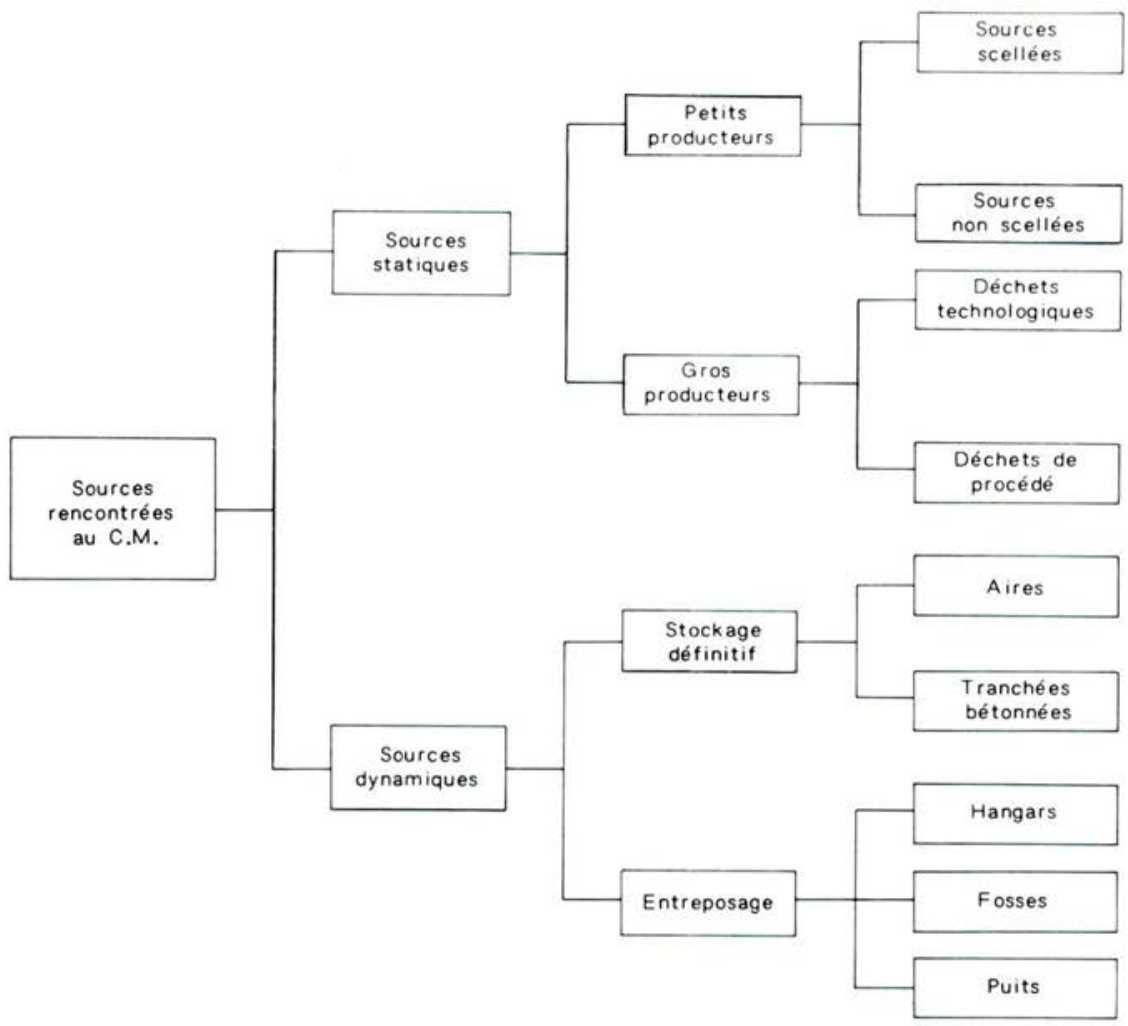

Fig. 2. - Les différents types de sources rencontrées au Centre de la Manche (CM).

\section{II.2. Caractéristiques des sources}

\section{II.2.1. Sources statiques}

Les caractéristiques des sources statiques reçues au CM sont largement définies par les règles édictées par l'ANDRA (voir figure 1) et par l'origine des déchets.

Les déchets provenant des petits producteurs présentent les caractéristiques suivantes [6]:

- Sources scellées : période relativement courte et activité assez faible, les sources de très forte activité étant généralement reprises par les fournisseurs pour réutilisation éventuelle. (A noter l'opération particulière d'assainissement menée par le Service central de protection contre les rayonnements ionisants (SCPRI) concernant la récupération des sources de radium 226). 
- Sources non scellées : ces produits ont souvent subi des opérations de dilution plus ou moins importantes. Ils présentent, en général, des périodes courtes, une faible radiotoxicité et une activité proche de la limite inférieure d'activité massique $\left(2.10^{-9}\right.$. Ci. . $\left.^{-1}\right)$ d'un produit considéré comme radioactif. Les radionucléides les plus fréquents sont: ${ }^{241} \mathrm{Am}$, ${ }^{137} \mathrm{Cs}, \quad{ }^{14} \mathrm{C}, \quad{ }^{60} \mathrm{Co},{ }^{57} \mathrm{Co}, \quad 55 \mathrm{Fe},{ }^{3} \mathrm{H}, \quad{ }^{125} \mathrm{I}, 192 \mathrm{Ir},{ }^{63} \mathrm{Ni}$, $210 \mathrm{~Pb}, \quad 22 \mathrm{Na}, 90 \mathrm{Sr},{ }^{204} \mathrm{TI},{ }^{226} \mathrm{Ra}, \mathrm{U}$ nat, ${ }^{239} \mathrm{Pu}$. Ils se situent dans la gamme des très faibles activités volumiques $(<1000 \mathrm{CMA})$.

Les déchets provenant des gros producteurs sont les plus importants en volume, les déchets technologiques représentant la plus forte proportion. Ils ont pour origine l'ensemble des installations nucléaires. Ils sont associés aux travaux d'entretien, de contrôle et d'assainissement de circuits, d'appareils ou d'installations. Ils se présentent donc sous des formes très variées. Ils sont constitués de toutes les matières utilisées dans les installations. Les radionucléides contenus sont très divers tant par leur mode de formation (fission, activation, transmutation) que par leurs caractéristiques : gamme étendue de la valeur de la période, toutes les radiotoxicités, radioactivité très faible à moyenne, toute la gamme des rayonnements avec des énergies très variées.

Les déchets de procédé ont des origines et des natures plus spécifiques et plus constantes. Ils sont généralement associés à des opérations industrielles et se situent essentiellement dans la catégorie "moyenne activité".

Les radionucléides rencontrés couvrent également une gamme importante, mais leurs caractéristiques présentent des domaines de variations plus restreints que ceux des déchets technologiques. Les déchets de procédé prendront peu à peu une part plus importante des volumes collectés.

En 1980 , le Centre a reçu environ 80000 colis ou sources statiques.

\subsubsection{Les sources dynamiques}

Les caractéristiques de ces sources sont liées au principe de stockage retenu. Si le stockage est définitif, la source est constituée d'un empilement judicieux des sources statiques précédentes noyées au fur et à mesure de l'avancement de la structure:

- dans du tout venant de carrière, du sáble, de l'argile, etc. si le stockage se fait sur aire :

- dans du béton, mortier ou coulis agréé si le stockage se fait en TB.

La structure est donc évolutive et le critère de réalisation est l'occupation maximale des volumes compatibles avec la capacité radiologique de la structure achevée. La tranchée terminée est un monobloc de béton constitué de parallélépipèdes élémentaires (pas d'avancement). Les tranchées bétonnées sont réalisées en-dessous du niveau normal des terres. Après achèvement, on réalise par dessus un stockage sur aire.

Pour chaque type de structure, l'activité cumulée croît tout au long de la réalisation pour être maximale une fois la structure achevée, mais le champ de rayonnements doit rester inférieur aux valeurs réglementaires tout au 
long de la réalisation du stockage. Si le principe retenu est l'entreposage, la structure d'accueil des sources est calculée et réalisée pour permettre une reprise possible ou obligatoire sans contraintes majeures d'irradiation du personnel. La source est achevée lorsque la capacité d'accueil est atteinte.

Toutes les structures de stockage sont décrites plus en détail dans l'analyse des postes de travail.

\section{LES POSTES DE TRAVAIL ET LES EXPOSITIONS ASSOCIES}

Les différents postes de travail que nous pouvons trouver sur le CM, sont regroupés sur le diagramme de la figure 3 .

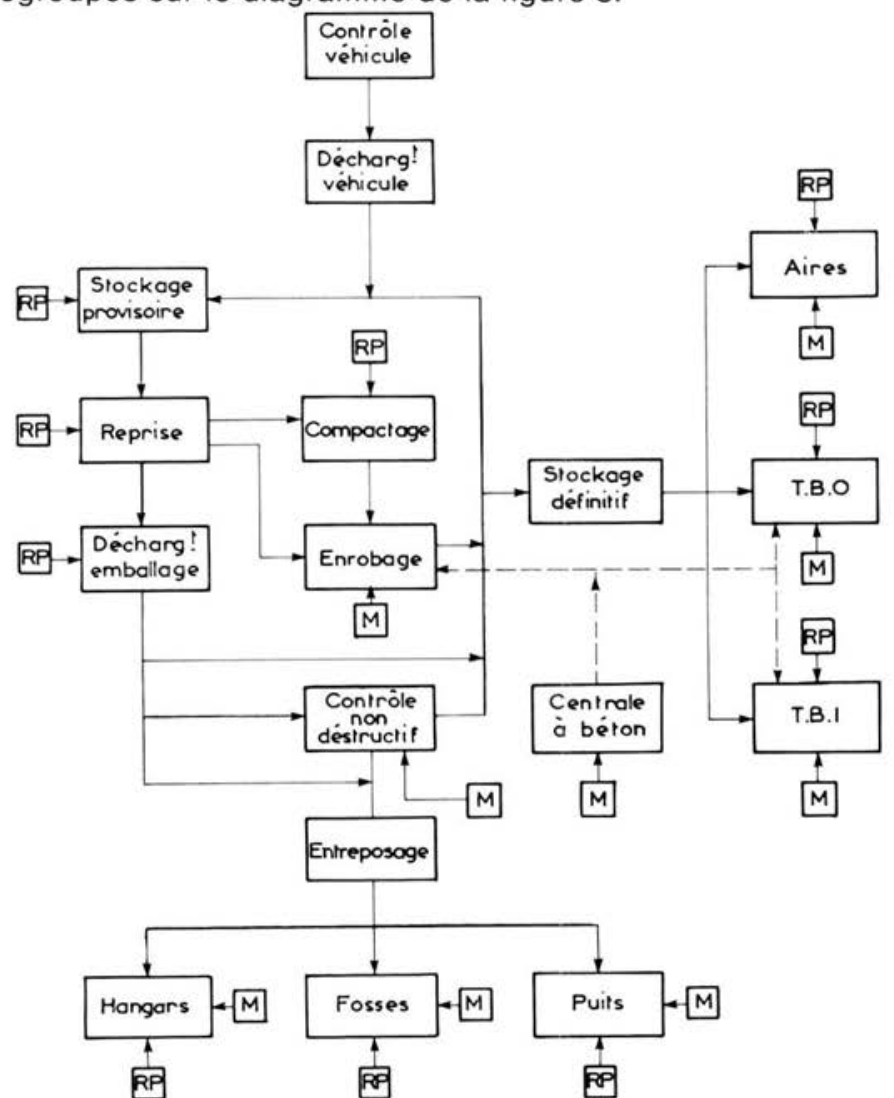

Fig. 3. - Postes de travail et radioprotection au CM.

$$
\begin{array}{ll}
\text { RP : Radioprotection } & \text { T.B.O. : Tranchée bétonnée ordinaire } \\
\text { M : Manutention } & \text { T.B.I. : Tranchée bétonnée irradiante }
\end{array}
$$


On peut distinguer successivement :

- les postes liés au conditionnement de certains déchets et à la réalisation de certaines structures (compactage, enrobage, centrales à béton, mortier ou coulis) ;

- les postes liés au stockage définitif (aires et tranchées bétonnées) ;

- les postes liés à l'entreposage (hangars, fosses, puits) ;

- les postes liés aux manutentions;

- les postes liés à la radioprotection.

\section{III.1. Les postes de travail liés au conditionnement des déchets et à la réalisation de certaines structures}

Une des prescriptions de stockage est de ne pas modifier le conditionnement des déchets arrivant sur le centre. Cependant, certains déchets non enrobés de faible et moyenne activité en fût métallique ne sont pas stockés dans leur emballage primaire. Ils sont compactés puis placés en coque de béton et enrobés dans un coulis béton avant d'être stockés sur aire. Ce nouveau conditionnement a pour but de réduire les volumes, afin d'optimiser le stockage tout en améliorant la sûreté, mais surtout d'assurer un confinement supplémentaire par enrobage.

\section{III.1.1. Compactage, enrobage}

Le compactage de certains déchets non enrobés est effectué par lot de 10 fûts de 200 I compressés successivement à l'aide d'une presse de $400 \mathrm{t}$, placée en enceinte ventilée. L'entrée des fûts et matériaux et la sortie des blocs se font par sas étanches évitant tout risque de contamination par aérosols et poussières.

Les fûts sont compactés dans un cylindre d'acier, vidés ensuite dans une coque en béton de $1,40 \mathrm{~m}$ de diamètre et de $1,30 \mathrm{~m}$ de hauteur. Le remplissage du bloc est complété, après dépose d'un carré de béton, par un coulis de béton avec scellement d'un couvercle. La presse est commandée à distance depuis un pupitre. Béton, mortier ou coulis sont injectés directement par pompe pneumatique depuis la centrale à béton. En fonctionnement normal, ces postes de travail sont peu irradiants, mais présentent des risques de contamination d'où un contrôle permanent de non contamination des lieux de travail. Par contre, les opérations d'intervention présentent ces deux risques associés. Ces postes de travail sont servis par trois agents travaillant en équipe.

\section{III.1.2. Centrales à béton}

Le centre est équipé de deux centrales à béton : d'une part pour la réalisation de l'enrobé de certains déchets, d'autre part pour obtenir les bétons, mortiers ou coulis nécessaires à la réalisation des tranchées bétonnées. Dans ce dernier cas, l'enrobé est amené sur le chantier par autotransporteur à béton. Ces postes de travail sont des postes "froids", mais 
ils sont essentiels au bon fonctionnement du centre. Le nombre d'agents affectés à ces tâches varie en fonction du planning des réalisations de structures ou des éléments annexes à ces structures.

\section{III.2. Postes de travail liés au stockage définitif (fig. 3)}

\section{III.2.1. Les aires de stockage}

Les aires ou plates-formes sont réalisées au niveau du sol ou au-dessus de certaines tranchées bétonnées. Elles reçoivent des blocs sur 4 hauteurs $(5,10 \mathrm{~m})$, qui constituent les structures périphériques. Les fûts métalliques, les filtres et les caissons sont judicieusement disposés à l'intérieur. Le remplissage des vides est fait avec des graviers. La plate-forme terminée, le terrain est aménagé avec du tout venant de carrière, compacté sur une épaisseur de $20 \mathrm{~cm}$ et avec une pente de $0,05 \mathrm{~m} / \mathrm{m}$ avant apport d'un revêtement imperméable. La réalisation de cette structure fait appel presque exclusivement à des opérations de manutention avec grue et, pour mise en place des déchets, engins automoteurs, camions, bulls, etc., pour les matériaux de remplissage et de finition. Le but final est d'amener l'aire achevée à ne présenter que des débits d'équivalent de dose proches de ceux du sol naturel avoisinant.

Les opérations les plus irradiantes sont la manutention des fûts pour leur mise en place et le remplissage des vides interstitiels.

\section{III.2.2. Les tranchées bétonnées}

Elles sont de deux types:

- les tranchées bétonnées ordinaires ou TBO ;

- les tranchées bétonnées irradiantes ou TBI.

TBO - Elles sont creusées dans le sol avec réalisation d'un radier de base drainé. Sur ce radier, sont déplacées des banches de coffrage qui forment une case élémentaire dans laquelle sont déposés les déchets. Ceux-ci sont ensuite noyés par couches dans le béton, afin de constituer un monolithe. La tranchée se réalise à l'avancement, case par case. Toutes les cases réalisées, la tranchée est terminée par réalisation d'une couverture bitumineuse. La dépose des déchets est l'opération la plus irradiante.

TBI - Ce sont des couloirs ménagés entre deux monolithes de TBO, destinés à recevoir les déchets irradiants de moyenne activité $\left(\mathrm{D}>0,1 \mathrm{~Gy} \cdot \mathrm{h}^{-1}\right)$. La dépose des déchets se fait à l'aide d'un château cloche avec operculaire et train de dalles de couverture associés, assurant une continuité de protection contre l'irradiation. Les fûts sont empilés dans une armature métallique. Lorsque la hauteur limite est atteinte, le tout est noyé dans le béton. L'avancement se fait pas à pas. Ce type de tranchée ne doit pas poser de problèmes d'irradiation, la continuité de protection étant assurée à tous les stades de la réalisation. 


\section{III.3. Postes de travail liés à l'entreposage}

\section{III.3.1. Les puits de décroissance}

L'installation est un monolithe de béton enterré comprenant 170 puits de $8 \mathrm{~m}$ de profondeur et $0,396 \mathrm{~m}$ de diamètre. Chaque puits est fermé par un bouchon en béton lourd avec joint d'étanchéité. Un pont dessert chaque puits et le tout est abrité par un hangar fermé. La dépose des fûts de 50 ou 60 I de déchets dans un puits se fait par un château de transfert et operculaire associé. Là encore, il y a continuité de la protection contre l'irradiation tout au long des opérations. Cette installation reçoit des déchets d'activité spécifique $\alpha>2.10^{4}$ CMA. La reprise des déchets est obligatoire.

\section{III.3.2. Les fosses}

Ce sont des structures destinées à recevoir les déchets dont l'activité spécifique $\alpha$ est comprise entre $2.10^{4}$ et $2.10^{5} \mathrm{CMA}$ avec reprise possible ainsi que des déchets particuliers (sulfate de plomb radifères).

\section{III.3.3. Les hangars}

Ils sont destinés à un entreposage provisoire de déchets particuliers en attente de réalisation de structures de stockage définitif, ou avec reprise possible. Ils servent aussi à l'entreposage transitoire de déchets qui constituent un stock de régularisation du fonctionnement du $\mathrm{CM}$. Ce type de structure est employé le moins souvent possible, car, pour un même colis, il augmente le nombre des manutentions, donc la dose d'irradiation du personnel.

Enfin, mentionnons pour terminer ce paragraphe, certains stockages particuliers, tels que : le bouclier thermique du réacteur de $\mathrm{Chooz}$, une fosse spéciale pour stockage d'un générateur électrique au ${ }^{60} \mathrm{Co}$ (gicodyne).

\section{III.4. Les postes liés à la manutention}

Deux types de postes:

- les postes spécifiques qui concernent ie déchargement des véhicules, le stockage provisoire des déchets, leur reprise et le déchargement des emballages de tous types. Cette dernière opération est l'une des plus irradiantes, nous aurons l'occasion d'y revenir;

- des opérations de manutention liées à tous les autres postes déjà analysés. Toutes les manutentions sont effectuées avec des moyens mécaniques appropriés à l'opération à effectuer: clés à chocs pour dévisser les emballages, grues de levage, portiques, chariots élévateurs, tracteurs, bulldozers, basculeurs à béton, etc.

Tous ces moyens concourent à améliorer les conditions de travail et à réduire les expositions subies par le personnel (réduction des temps et augmentation de la distance source-opérateur, blindages continus). 


\section{III.5. Les postes liés à la radioprotection}

La radioprotection est présente à tous les niveaux du travail, depuis l'arrivée d'un véhicule sur le centre, jusqu'à la mise en place définitive du colis. On retrouve tous les contrôles réglementaires de radioprotection d'une INB :

- zone contrôlée et ses subdivisions ;

- contrôle du personnel (entrée et sortie de zone contrôlée), des structures (pendant et après réalisation), des diverses installations (presse, hangars, fosses, puits, etc.), des matériels et du site (contrôles atmosphériques, eaux de ruissellement et eaux du réseau séparatif) ;

- la dosimétrie individuelle et la dosimétrie de zone ;

- les contrôles périodiques des matériels de radioprotection.

Certaines mesures relatives à ces contrôles sont effectuées contractuellement par la COGÉMA.

II faut mentionner également une installation spécifique de contrôle non destructif par spectrométrie $\alpha$ des déchets (réputés $\alpha$ ).

La mesure de la teneur des fûts se fait par diode germanium-lithium. Cette installation est quasi opérationnelle. L'arrivée, l'analyse et le départ des fûts se font en séquentiel. Cette installation ne donne pas lieu à irradiation du personnel sauf pour son approvisionnement en fûts de déchets.

\section{LES RESULTATS DOSIMETRIQUES ET LEUR ANALYSE}

Le CM est dans sa $12^{e}$ année d'exploitatıon. Nous disposons donc des résultats de la dosimétrie individuelle du personnel portant sur 11 années consécutives. Ils sont résumés sur la figure 4 où nous avons tracé :

- l'équivalent de dose collectif annuel (organisme entier) ;

- l'équivalent de dose annuel (organisme entier) de l'agent le plus irradié ;

- l'équivalent de dose moyen annuel individuel (organisme entier) ;

- le volume annuel de déchets réceptionnés au CM. 


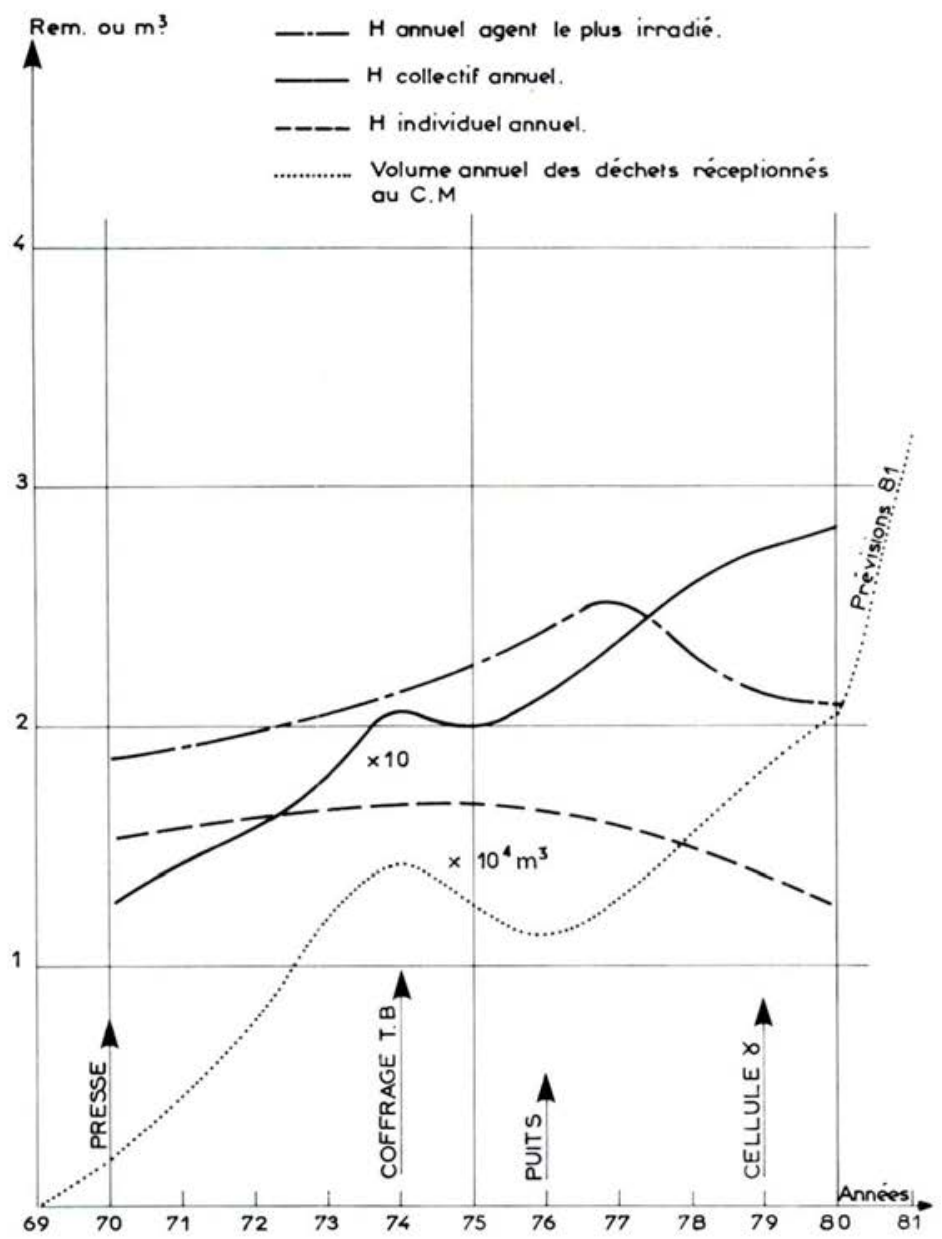

Fig. 4. - Résultats dosimétriques de 11 années d'exploitation au CM.

Que remarquons-nous?

- Entre 1973 et 1980 , le volume des déchets reçus au CM a été multiplié par 4. Actuellement, l'augmentation annuelle est de l'ordre de 50 à 60 p. cent.

- L'équivalent de dose collectif annuel suit sensiblement la même progression que le volume des déchets jusqu'au milieu de 1978 où une tendance au ralentissement s'amorce. En 1980, la dose collective annuelle est évaluée à environ 0,3 homme. Sv, soit $3,810^{-6}$ homme.Sv par colis reçu. II faut attendre fin 1981 pour vérifier si cette tendance se précise.

- L'équivalent de dose moyen annuel individuel est en diminution constante depuis 1974-1975. Ceci est la résultante de plusieurs actions : 
mise en œuvre des coffrages pour la réalisation des TB et amélioration permanente des techniques de réalisation,

accroissement et renouvellement du parc des engins mécaniques de levage et de manutention avec rationalisation des opérations à effectuer,

action de formation et d'information de tout le personnel dès son embauche : stages d'initiation à la radioprotection, de cariste, de grutier, de pontier élingueur, etc.

- L'équivalent de dose annuel individuel est en nette réduction depuis la parution, en 1976, d'un cahier des charges définissant de façon stricte, les règles auxquelles doit satisfaire tout colis agréé.

\section{TENDANCES ET PERSPECTIVES}

II serait tentant de dire que tout ne marche pas trop mal au CM sur le plan dosimétrique, et cela est vrai. Mais je préfère conclure en parlant des améliorations qui sont en cours et ce qui est envisagé.

Les opérations de manutention sont très nombreuses et génératrices de doses d'exposition intégrées. Le souci permanent est de réduire leur nombre et la nuisance, en les mécanisant au maximum. Chaque fois que nous ne pouvons plus bénéficier de l'écran de l'emballage, nous jouons sur le temps et la distance par l'utilisation d'outils ou d'engins dont les performances sont adaptées aux colis à traiter. Mais, l'effort le plus important au niveau des investissements est consenti à la protection collective sans toutefois négliger la protection individuelle. Ces investissements concernent : les études d'amélioration de solutions de continuité dans les moyens de protection, depuis l'arrivée du colis jusqu'à sa mise en place en structure de stockage, la réalisation des équipements nécessaires à cette continuité.

Les TBI qui vont bientôt entrer en service sont un exemple de cette continuité. Les déchets irradiants de moyenne activité seront repris directement de l'emballage de transport à l'aide d'un château cloche de reprise, et descendus directement dans un des puits réalisés à cet effet. II n'y aura donc ni rupture de confinement, ni rupture de la protection biologique. Des TBTI sont déjà à l'étude pour le stockage des déchets de débit de dose supérieure à $0,1 \mathrm{~Gy} \cdot \mathrm{h}^{-1}$ au contact du colis.

Mais ceci m'amène à parler de la réglementation des transports. Les expositions moyennes du personnel affecté au transport industriel des déchets demeurent à des valeurs basses : $2,5 \mathrm{mSv}(250 \mathrm{mrem})$ pour l'année 1980 et pour le personnel réellement exposé. Ces résultats correspondent à $25000 \mathrm{t}$ de déchets radioactifs transportés sur un parcours total de $850000 \mathrm{~km}$, soit environ $2.10^{10} \mathrm{t} . \mathrm{km}$.

Tous ces transports ont pour terminal le CM. II y a donc accumulation des colis en un point unique où toutes les doses nécessaires à leur déchargement vont être cumulées par un nombre restreint de personnes. C'est un problème important qui ne semble pas avoir été pris en compte dans la mise en œuvre des principes de sûreté. L'emballage lui-même n'a pas 
toujours été conçu pour un accès rapide au contenu, et son entretien n'est pas toujours effectué. Les débits de dose tolérés au contact de la paroi extérieure de l'emballage conduisent certainement à la part la plus importante de la dose intégrée par le personnel des centres de stockage de déchets solides.

Je m'adresse donc aux producteurs de déchets et aux propriétaires d'emballages, afin qu'ils soient conscients des problèmes qui peuvent se poser au niveau d'un centre national. Leurs propres problèmes sont multipliés par 10 ou 100, à l'arrivée au centre, sachant que, et ce sera une donnée de conclusion, le volume de déchets reçus au CM, en 1980, revient à traiter un colis toutes les 90 secondes. Multiplier le personnel ne peut qu'augmenter la dose collective, sans pour autant résoudre les problèmes.

\section{BIBLIOGRAPHIE}

[1] AGENCE NATIONALE POUR LA GESTION DES DECHETS RADIOACTIFS (ANDRA). Catalogue des colis agréés, édition 1981. Note ANDRA / 81-3248, 1981.

[2] Principes généraux de protection contre les rayonnements ionisants, décret n ${ }^{\circ} 66-450$ du 20 juin 1966, J.O.R.F., 30 juin 1966. Paris : Journaux officiels, 1966 (Fascicule $\left.n^{\circ} 66-76\right)$.

[3] Protection des travailleurs contre les dangers des rayonnements ionisants, décret $n^{\circ}$ 67-228 du 15 mars 1967, J.O.R.F., 22 mars 1967. Paris : Journaux officiels, 1967 (Fascicule n०67-42).

[4] Protection des travailleurs contre les dangers des rayonnements ionisants dans les installations nucléaires de base, décret $n^{\circ} 75-306$ du 28 avril 1975, J.O.R.F., 30 avril 1975. Paris : Journaux officiels, 1975 (Fascicule $n^{\circ} 75 / 87$ ).

[5] Transport et manutention des matières dangereuses : transport des matières radioactives (classe IV b), arrêté du 24 juin 1974 codifié le 8 août 1980. Paris : Journaux officiels, 1980 (Fascicule $n^{\circ} 74-28$ bis).

[6] SCHEIDHAUER J. - La collecte et le transport des déchets radioactifs de faible et moyenne activité. Séminaire sur la gestion des déchets radioactifs, Cadarache, 2.6 février 1981. 